\title{
Estimating Circular Distance in Graph Nodes
}

\section{J. Veeranjaneyulu, Varma. P. L. N.}

Abstract: In the current article we propose the concept of circular D-distance between nodes which is sum of Ddistance and detour D-distance. We show that this distance is a metric. Also we study some properties. Further we compute the circular D-distance of some classes of graphs and show that some of them are circular D-self-centered.

Keywords: circular D-distance, circular Dradius, circular D-diameter.Article Type: Research Article

\section{INTRODUCTION}

Let $r, s \in H$ be any two nodes of a graph $H$. The (standard) distance $d(r, s)$ within $r$ and $s$ is the length of the minimal $r-s$ path in $H$. Chartrand el al (see [2], [3]) proposed the new thought of longest distance in graphs as follows: For two nodes $r, s$ in a graph $H$, the longest distance $D(r, s)$ is defined as the length of the longest $r$ - $S$ path in $H$.

In [5,6], for any two nodes $r, s$ in a graph $H$, the $D$-length of a $r-S$ path was defined by considering degree of each node present in the path in addition to the length of the path. Using this length, Reddy Babu and Varma (in [5]), Venkateswara Rao and Varma (in [6]) defined the concept of $D$-distance and detour $D$-distance, respectively, as the minimum and maximum $D$-path lengths, respectively.

In this article we introduce a new distance, which we call as circular $D$-distance, within any two nodes of a graph $H$ by taking sum of $D$-distance and detour $D$ distance and study some of its properties. This distance is significantly different from other distances.

Similarly one can talk about the circular distance using distance and detour distance. In logistical management the circular distance plays a vital role. For instance, the goods delivery man wants to distribute the goods, during distribution of goods from a home place which is in city ' $L$ ' to a destination which is in another city ' $M$ ' covering all the places on the way. To come back home place, the shortest route may be selected so as to decrease the time, fuel consumption and vehicle cost to deliver the goods.

Revised Manuscript Received on December 15, 2019

J. Veeranjaneyulu, Division of Mathematics, Department of S\&H, Vignan's Foundation for Science, Technology \& Research,Vadlamudi522213, Guntur, India,

Varma. P. L. N.,Division of Mathematics, Department of S\&H, Vignan's Foundation for Science, Technology \& Research,Vadlamudi-522213, Guntur, India,

(E-mail: jangam.vfstr@gmail.com, E-mail: plnvarma@gmail.com).
In a forthcoming article [7] circular D-distance and path graphs will be studied. Throughout the article we consider non-trivial, finite, undirected, simple and connected graphs. For any notations and terminology we refer the book [1].

\section{CIRCULAR D-DISTANCE}

In this part we propose the concept of circular distance and circular $D$-distance. For reader convenience we recall some previous definitions.

Definition 2.1 (See [5]): Suppose $r, s$ are nodes of a graph $H$ and $S$ is a $r-S$ path. Then the D-length of the path $S, \quad$ is stated as $l^{D}(S)=l(S)+d(r)+d(s)+\sum d(t)$.

Definition 2.2 (See [5], [6]): If $r, s$ are any two nodes of a graph $H$, the $D$-distance between them is stated as $d^{D}(r, s)=\min \left\{l^{D}(S)\right\}$, where the minimal path is taken overall $r-s$ paths. if $r$ and $s$ are distinct and $d^{D}(r, s)=0$ if $r=s$. Similarly, the detour D-distance, $D^{D}(r, s) \quad$ between $r, s$ is stated as $D^{D}(r, s)=\max \left\{l^{D}(S)\right\}$ if $r$ and $s$ are distinct and $D^{D}(r, s)=0$ if $r$ and $s$ are identical where the maximum is taken over all $r-s$ paths.

Now we introduce the concepts of circular distance and circular $D$-distance within nodes of a graph as below:

Definition 2.3: If $r, s$ are any two nodes of a graph $H$, the circular distance within them is defined as the sum of distance $d(r, s)$ and detour distance $D(r, s)$. Here $d(r, s)$ is the length of geodesic path and $D(r, s)$ the length of the longest path within $r$ and $s$. This distance is denoted by $\operatorname{cir}(r, s)$.

Definition 2.4: The circular D-distance, $\operatorname{cir}^{D}(r, s)$, within two nodes $r, s$ of $H$ is stated as sum of $D$-distance and detour $D$-distance, In otherwords

$\operatorname{cir}^{D}(r, s)=\left\{\begin{array}{cc}\min \left\{l^{D}(S)\right\}+\max \left\{l^{D}(S)\right\} & \text { if } r \neq s \\ 0 & \text { if } r=s\end{array}\right.$

where the minimum and maximum are taken over all $r-s$ paths $S$ in $H$.

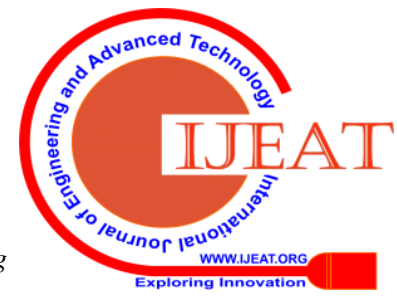




\section{SOME RESULTS ON CIRCULAR D-DISTANCE}

Here, we study some properties of circular $D$-distance. We begin with an important result. Theorem 3.1: For any graph $H$, the circular $D$-distance defines a metric on the set of all nodes.

Proof: Let $r, s, t$ be any three nodes of $H$. Then from the definition it is clear that $\operatorname{cir}^{D}(r, s) \geq 0$ and $\operatorname{cir}^{D}(r, s)=0 \Leftrightarrow r=s$. Also we have, $\operatorname{cir}^{D}(r, s)=\operatorname{cir}^{D}(s, r)$ and hence the circular $D$-distance is symmetric.

Next we have to show that the circular $D$-distance contents the triangular inequality. This essentially follows from the property that $D$-distance and detour $D$-distance are metrics on $H$. (See theorem 2.6 of [5] and theorem 3.1 of [6].). Let $r, t, s$ be any three nodes of $H$. Now $\operatorname{cir}^{D}(r, t)=d^{D}(r, t)+D^{D}(r, t)$ and $\operatorname{cir}^{D}(t, s)=d^{D}(t, s)+D^{D}(t, s)$. Thus

$$
\begin{aligned}
\operatorname{cir}^{D}(r, t)+\operatorname{cir}(t, s)=\left\{d^{D}(r, t)+D^{D}(r, t)\right\} & \left\{d^{D}(t, s)+D^{D}(t, s)\right\} \\
& =\left\{d^{D}(r, t)+d^{D}(t, s)\right\}+\left\{D^{D}(r, t)+D^{D}(t, s)\right\} \\
& \geq d^{D}(r, s)+D^{D}(r, s) \\
& \geq \operatorname{cir}^{D}(r, s)
\end{aligned}
$$

Thus $\operatorname{cir}^{D}(r, t)+\operatorname{cir}^{D}(t, s) \geq \operatorname{cir}^{D}(r, s)$, which shows that the triangular inequality is satisfied. Hence the circular $D$-distance contented a metric on the set of all nodes of $H$.

Next we introduce some terminology.

Definition 3.2: The maximum circular $D$-distance to any other node from a node $s$ is the circular $D$ eccentricity of a $s$ and is represented by $e_{C}^{D}(s)$. Thus $e_{C}^{D}(s)=\max \left\{\operatorname{cir}^{D}(r, s): r \in V(H)\right\}$.

Definition 3.3: A node $r$ for which $\operatorname{cir}^{D}(r, s)=e_{C}^{D}(s)$ is called circular D-eccentric node of $s$. If $s$ is the circular $D$-eccentric node of some node then it is called circular D-eccentric node of $H$.

Definition 3.4: The circular D-radius of $H$ is $r_{C}^{D}(H)=\min \left\{e_{C}^{D}(r): r \in V(H)\right\}$ and the circular $D$ diameter of $H$ is $d_{C}^{D}(H)=\max \left\{e_{C}^{D}(r), r \in V(H)\right\}$.

Definition 3.5: In a graph $H$, the circular D-center (circular D-periphery) is the subgraph induced by all nodes of $H$ of minimal (maximal) circular $D$-eccentricity. They are represented by $c_{C}^{D}(H)$ and $p_{C}^{D}(H)$ respectively.

Definition 3.6: If circular $D$-center of $H$ is same as the graph $H$, then it is called circular $D$-selfcentered. Alternatively, if circular $D$-radius and circular $D$-diameter are same then graph $H$ is circular $D$-self-centered.

Remark 3.7: For all the nodes of the center, the eccentricity is same as the radius in a graph.

Proposition 3.8: Let $r, s \in V(H)$. Then $\left|e_{C}^{D}(r)-e_{C}^{D}(s)\right| \leq \operatorname{cir}^{D}(r, s)$.

Proof: Given two nodes $r, s \in H$, choose a node $t$ of $H$ such that $\operatorname{cir}^{D}(r, t)=e_{C}^{D}(r)$.

Consider $e_{C}^{D}(r)=\operatorname{cir}^{D}(r, t) \leq \operatorname{cir}^{D}(r, s)+\operatorname{cir}^{D}(s, t)$ (from triangular inequality)

$$
\leq \operatorname{cir}^{D}(r, s)+e_{C}^{D}(s) \text {. }
$$

Thus $e_{C}^{D}(r)-e_{C}^{D}(s) \leq \operatorname{cir}^{D}(r, s)$. Hence $\left|e_{C}^{D}(r)-e_{C}^{D}(s)\right| \leq \operatorname{cir}^{D}(r, s)$.

\section{CIRCULAR D-DISTANCE OF SOME CLASSES OF GRAPHS}


Here, we compute the circular $D$-radius and circular $D$-diameter of some classes of graphs. We begin with the class of complete graph.

Theorem 4.1: Let $K_{m}$ be the complete graph with $m$ nodes $(m \geq 3)$. Then the circular $D$-eccentricity of any node is $m^{2}+2 m-2$. Thus we have $r_{C}^{D}\left(K_{m}\right)=d_{C}^{D}\left(K_{m}\right)=m^{2}+2 m-2$.

Proof: Let $K_{m}$ be a complete graph with $m$ nodes $(m \geq 3)$. In this graph each node is of degree $m-1$. Any two nodes are adjacent and hence minimal path length is 1 and detour path length is $m-1$.

From the definition of circular $D$-distance, for any two nodes $r, s$ we have

$$
\begin{aligned}
\operatorname{cir}^{D}(r, s)= & d^{D}(r, s)+D^{D}(r, s) \\
= & \{1+(\text { sum of the degrees of all nodes in } r-s \text { path })\}+ \\
& \quad\{m-1+(\text { sum of the degrees of all nodes in detour } r-s \text { path })\} \\
= & \{1+2(m-1)\}+\{m-1+m(m-1)\} \\
= & m^{2}+2 m-2 .
\end{aligned}
$$

Thus circular $D$-eccentricity of each node is $m^{2}+2 m-2 . r_{C}^{D}\left(K_{m}\right)=d_{C}^{D}\left(K_{m}\right)=m^{2}+2 m-2$.

Corollary 4.2: The complete graph $K_{m}$ is circular D-self-centered.

Next we discuss the classes of cyclic graphs.

Theorem 4.3: Let $C_{n}$ be the cyclic graph with $n$ nodes. Then the circular $D$-distance within any two nodes is $3 n+4$. Thus we have $d_{C}^{D}\left(C_{n}\right)=r_{C}^{D}\left(C_{n}\right)=3 n+4$.

Proof: Let $r_{1}, r_{2}, \cdots, r_{i}, r_{j}, \cdots, r_{n}$ be nodes of the cyclic graph $C_{n}$. In this graph degree of each node is 2 . Within any two nodes $r_{i}$ and $r_{j}$ there are two paths. If one of them has $k$ edges, the other would contain $n-k$ edges. Clearly one of them in a geodesic path and other will be longest path. Since the $D$-distance is calculated along the shortest path and detour $D$-distance is calculated along the longest path, in calculating circular $D$-distance, we need to consider both the paths. Thus $\operatorname{cir}^{D}\left(r_{i}, r_{j}\right)=d^{D}\left(r_{i}, r_{j}\right)+D^{D}\left(r_{i}, r_{j}\right)$. The sum on the right hand side consists of both the paths and the degrees of all nodes with $d\left(r_{i}\right), d\left(r_{j}\right)$ repeating. Thus $\operatorname{cir}^{D}\left(r_{i}, r_{j}\right)=k+(n-k)$

$+2 n+2+2=3 n+4$. Thus observe that $\operatorname{cir}^{D}\left(r_{i}, r_{j}\right)=3 n+4$ for every $r_{i}, r_{j} \in V\left(C_{n}\right)$. Therefore $e_{C}^{D}\left(r_{i}\right)=3 n+4$. Thus $d_{C}^{D}\left(C_{n}\right)=r_{C}^{D}\left(C_{n}\right)=3 n+4$.

Corollary 4.4: The cyclic graph $C_{n}$ is circular $D$-self-centered.

Next we consider the wheel graph. Under this we have

Theorem 4.5: Let $W_{1, n}$ be wheel graph with $n+1$ nodes. We have $r_{C}^{D}\left(W_{1, n}\right)=6 n+4$ and $d_{C}^{D}\left(W_{1, n}\right)=6 n+8$ for $n \geq 6$. Further we have $r_{C}^{D}\left(W_{1,3}\right)=d_{C}^{D}\left(W_{1,3}\right)=22, r_{C}^{D}\left(W_{1,4}\right)=28, d_{C}^{D}\left(W_{1,4}\right)=31$, $r_{C}^{D}\left(W_{1,5}\right)=34, d_{C}^{D}\left(W_{1,5}\right)=36$.

Proof: Let $W_{1, n}$ be the wheel graph with nodes $\left\{r_{0}, r_{1}, \cdots, r_{n}\right\}$ where $r_{0}$ is adjacent to all other nodes and the degrees of another nodes are 3. For $n=3, \operatorname{cir}^{D}\left(r_{i}, r_{j}\right)=22$. Then the circular $D$-eccentricity of each node is 22 . Thus circular $D$-radius and circular $D$-diameter of $W_{1,3}$ is 22 , so $W_{1,3}$ is selfcentered.

For $n=4, \operatorname{cir}^{D}\left(r_{0}, r_{j}\right)=28$ and $\operatorname{cir}^{D}\left(r_{i}, r_{j}\right)=27$ or 31 if $i \neq 0$ for all $j$. Thus the circular $D$ eccentricity of $r_{0}$ is 28 and circular $D$-eccentricity of other nodes is 31 . Thus circular $D$-radius of $W_{1,4}$ is 28 and circular $D$-diameter of $W_{1,4}$ is 31 . 
For $n=5, \operatorname{cir}^{D}\left(r_{0}, r_{j}\right)=34$ and $\operatorname{cir}^{D}\left(r_{i}, r_{j}\right)=32$ or 36 if $i \neq 0$ for all $j$. Thus the circular $D$ eccentricity of $r_{0}$ is 34 and circular $D$-eccentricity of other nodes is 36 . Thus circular $D$-radius of $W_{1,5}$ is 34 and circular $D$-diameter of $W_{1,5}$ is 36 .

For a wheel graph $W_{1, n}$ with $n \geq 6$ and $d\left(r_{0}\right)=n$ and all remaining $n$ nodes have degree 3 . Let $r_{0}, r_{i}, r_{j} \in W_{1, n}$. Then $\operatorname{cir}^{D}\left(r_{0}, r_{j}\right)=d^{D}\left(r_{0}, r_{j}\right)+D^{D}\left(r_{0}, r_{j}\right)$

$$
\begin{aligned}
= & {\left[1+d\left(r_{0}\right)+\text { sum of degrees of } W_{1, n} \text { in } r_{0}-r_{j} \text { path }\right]+} \\
& {\left[n+d\left(r_{0}\right)+\text { sum of deg rees of } W_{1, n} \text { in } r_{0}-r_{j} \text { path }\right] } \\
= & {[1+n+3+n+n+3 n] } \\
= & 6 n+4
\end{aligned}
$$

Thus $e_{C}^{D}\left(r_{0}\right)=6 n+4$.

$$
\begin{aligned}
\operatorname{cir}^{D}\left(r_{i}, r_{j}\right)= & d^{D}\left(r_{i}, r_{j}\right)+D^{D}\left(r_{i}, r_{j}\right) \\
= & {\left[2+d\left(r_{0}\right)+2\left(\text { sum of degrees of } W_{1, n} \text { in } r_{i}-r_{j} \text { path }\right)\right]+} \\
& {\left[n+d\left(r_{0}\right)+\text { sum of degrees of } W_{1, n} \text { in } r_{i}-r_{j} \text { path }\right] } \\
= & {[2+n+2 * 3+n+n+3 n] } \\
= & 6 n+8
\end{aligned}
$$

Thus $e_{C}^{D}\left(r_{i}\right)=6 n+8$. Now $e_{C}^{D}\left(r_{i}\right)>e_{C}^{D}\left(r_{0}\right)$. Hence $r_{C}^{D}\left(W_{1, n}\right)=6 n+4 . d_{C}^{D}\left(W_{1, n}\right)=6 n+8$.

Theorem 4.6: (i) Let $K_{x, y}$ be the complete bipartite graph with $x+y$ nodes, where $x<y$.

Then

if $y \geq 3 x+2$ then $r_{C}^{D}\left(K_{x, y}\right)=x(x+5)+y(x+1)+2, d_{C}^{D}\left(K_{x, y}\right)=x^{2}+x y+2(x+y)$.

if $y \leq 3 x+2$ then $r_{C}^{D}\left(K_{x, y}\right)=x^{2}+x y+2(x+y), d_{C}^{D}\left(K_{x, y}\right)=x(x+5)+y(x+1)+2$.

(ii) In the complete bipartite graph, $K_{x, x}$, we have $r_{C}^{D}\left(K_{x, x}\right)=d_{C}^{D}\left(K_{x, x}\right)=2 x^{2}+4 x$.

Proof: In a complete bipartite graph $K_{x, y}$, the node set can be split into two sets $U_{1}$ and $U_{2}$ containing $x$ and $y$ nodes, respectively, such that each edge in $K_{x, y}$ will have one end node in $U_{1}$ and the other in $U_{2}$.

First we compute the circular $D$-distance between two nodes from $U_{1}$.

Let $r_{1}, s_{1} \in U_{1}$. Then for any $r_{2} \in U_{2}$ we have a path $r_{1}-r_{2}-s_{1}$ between $r_{1}$ and $s_{1}$

$$
\begin{aligned}
\operatorname{cir}^{D}\left(r_{1}, s_{1}\right)= & d^{D}\left(r_{1}, s_{1}\right)+D^{D}\left(r_{1}, s_{1}\right) \\
= & {\left[2+d\left(r_{1}\right)+d\left(s_{1}\right)+d\left(r_{2}\right)\right]+} \\
& {[2 x-2+\text { the degrees of all nodes of } U+x(x-1)] } \\
= & {[2+(y+y)+x]+[2 x-2+x y+x(x-1)] } \\
= & {[2+2 y+x+2 x-2+x y+x(x-1)] } \\
= & x^{2}+x y+2(x+y)
\end{aligned}
$$

Therefore $\operatorname{cir}^{D}\left(r_{1}, s_{1}\right)=x^{2}+x y+2(x+y) \cdots \cdots \cdots(1)$.

Similarly for any two nodes $r_{2}, s_{2} \in U_{2}$ we have $\operatorname{cir}^{D}\left(r_{2}, s_{2}\right)=x(x+5)+y(x+1)+2 \cdots \cdots \cdots(2)$.

Finally, we compute the circular $D$-distance between a node of $U_{1}$ and a node of $U_{2}$. 
Let $r_{1} \in U_{1}$ and $s_{2} \in U_{2}$. Then there is path $r_{1}-s_{2}$ in the graph. Then

$$
\begin{aligned}
\operatorname{cir}^{D}\left(r_{1}, s_{2}\right)= & d^{D}\left(r_{1}, s_{2}\right)+D^{D}\left(r_{1}, s_{2}\right) \\
= & {\left[1+d\left(r_{1}\right)+d\left(s_{2}\right)\right]+} \\
& {\left[(2 x-1)+\left(\text { sum of degrees of all nodes of } U_{1}\right)+x(x)\right] } \\
= & 1+x+y+2 x-1+x y+x(x) \\
= & x(x+3)+y(x+1)
\end{aligned}
$$

Therefore $\operatorname{cir}^{D}\left(r_{1}, s_{2}\right)=x(x+3)+y(x+1)$

From(3) - (2), we have $\operatorname{cir}^{D}\left(r_{1}, s_{2}\right)-\operatorname{cir}^{D}\left(r_{2}, s_{2}\right)=x^{2}+3 x+x y+y-x^{2}-5 x-x y-y-2=-2 x-2$ and this is always negative. Hence the eccentricity of nodes from $U_{2}$ is $x(x+5)+y(x+1)+2=\boldsymbol{a}($ say).

Similarly, from $(3)-(1)$, we have $\operatorname{cir}^{D}\left(r_{1}, s_{2}\right)-\operatorname{cir}^{D}\left(r_{1}, s_{1}\right)=x^{2}+3 x+x y+y-x^{2}-x y-2 x-2 y=x-y$ and this is always negative. Thus the eccentricity of nodes from $U_{1}$ is $x^{2}+x y+2(x+y)=\boldsymbol{b}$ (say).

To calculate the radius and diameter, let's compare these eccentricities.

If $y \geq 3 x+2$ then $a>b \quad$ and hence $r_{C}^{D}\left(K_{x, y}\right)=x(x+5)+y(x+1)+2, \quad d_{C}^{D}\left(K_{x, y}\right)=x^{2}+x y+$ $2(x+y)$. Similarly, if $y \leq 3 x+2$ then $a<b$ and hence $r_{C}^{D}\left(K_{x, y}\right)=x^{2}+x y+2(x+y)$, $d_{C}^{D}\left(K_{x, y}\right)=x(x+5)+y(x+1)+2$. Hence the theorem is proved.

ii) In a complete bipartite graph $K_{x, x}$ the node set can be divided into two sets $U_{1}$ and $U_{2}$ containing $x$ and $x$ nodes, respectively, such that each edge would have one end node in $U_{1}$ and the other in $U_{2}$.

First we compute the circular $D$ - distance between two nodes from $U_{1}$. Let $r_{1}, s_{1} \in U_{1}$. Then for any $r_{2} \in U_{2}$ we have path $r_{1}-r_{2}-s_{1}$ between $r_{1}$ and $s_{1}$. Then

$$
\begin{aligned}
\operatorname{cir}^{D}\left(r_{1}, s_{1}\right)= & {\left[2+d\left(r_{1}\right)+d\left(s_{1}\right)+d\left(r_{2}\right)\right]+} \\
& {\left[2 x-2+\text { the degrees of all nodes of } U_{1}+x(x-1)\right] } \\
= & {[2+(x+x)+x]+[(2 x-2)+x(x)+x(x-1)] } \\
= & {\left[2+3 x+2 x-2+x(x)+x^{2}-x\right] } \\
= & 2 x^{2}+4 x
\end{aligned}
$$

Therefore $\operatorname{cir}^{D}\left(r_{1}, s_{1}\right)=2 x^{2}+4 x \cdots \cdots \cdots(1)$.

Similarly for any two nodes $r_{2}, s_{2} \in U_{2}$ we have $\operatorname{cir}^{D}\left(r_{2}, s_{2}\right)=2 x^{2}+4 x \cdots \cdots \cdots(2)$.

Finally, we compute the circular $D$-distance between a node of $U_{1}$ and a node of $U_{2}$.

Let $r_{1} \in U_{1}$ and $s_{2} \in U_{2}$. Then there is path $r_{1}-s_{2}$ in the graph. Then

$$
\begin{aligned}
\operatorname{cir}^{D}\left(r_{1}, s_{2}\right) & =\left[1+d\left(r_{1}\right)+d\left(s_{2}\right)\right]+\left[(2 x-1)+\left(\text { degrees of all nodes of } U_{1}\right)+x(x)\right] \\
& =1+(x+x)+[(2 x-1)+(x) x+x(x)] \\
& =2 x^{2}+4 x
\end{aligned}
$$

Therefore $\operatorname{cir}^{D}\left(r_{1}, s_{2}\right)=2 x^{2}+4 x \cdots \cdots \cdots(3)$.

Thus the circular $D$-distance within any two nodes in $K_{x, x}$ is $2 x^{2}+4 x$.

Therefore, eccentricity of any node of $r$ in $K_{x, x}$ is $e_{C}^{D}(r)=2 x^{2}+4 x$

Hence $r_{C}^{D}\left(K_{x, x}\right)=d_{C}^{D}\left(K_{x, x}\right)=2 x^{2}+4 x$. Thus $K_{x, x}$ is a circular $D-$ selfcentered.

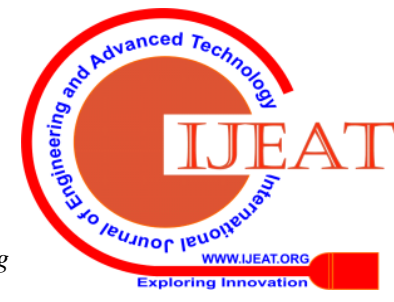




\section{RESULTS DESCRIPTION}

Remark: If $y=3 x+2$ then $r_{C}^{D}\left(K_{x, y}\right)=d_{C}^{D}\left(K_{x, y}\right)$.

Corollary: In a complete bipartite graph $K_{x, y}$ where $x<y$ we have $d_{C}^{D}\left(K_{x, y}\right)-r_{C}^{D}\left(K_{x, y}\right)=$ $3 x-y+2$. Therefore, if $y=3 x+2$, then this graph is circular $D-$ self-centered.

We end this section with the following proposition.

Proposition: In graph $H$, if circular distance and circular $D$-distance are obtained on the same path then we get $\operatorname{cir}^{D}(r, s)-\operatorname{cir}(r, s) \leq 4|E|$.

Proof: Let $H$ be connected graph such that circular distance and circular $D$-distance are obtained on the same path. i.e., in both distances, circular lengths are same but the difference is only degree of the nodes present on the path.

Consider

$$
\begin{gathered}
\operatorname{cir}^{D}(r, s)-\operatorname{cir}(r, s)=\min \left\{d(r, s)+\operatorname{deg}(r)+\operatorname{deg}(s)+\sum_{t \in r-\text { spath }} \operatorname{deg}(t)\right\}+ \\
\max \left\{D(r, s)+\operatorname{deg}(a)+\operatorname{deg}(s)+\sum_{t \in r-\text { spath }} \operatorname{deg}(t)\right\}-\operatorname{cir}(r, s) \\
\quad=\left\{d(r, s)+D(r, s)+2 \operatorname{deg}(r)+2 \operatorname{deg}(s)+2 \sum_{t \in r-s \text { path }} \operatorname{deg}(t)\right\}-\operatorname{cir}(r, s) \\
=\operatorname{cir}(r, s)+2 \operatorname{deg}(r)+2 \operatorname{deg}(s)+2 \sum_{t \in r-s \text { path }} \operatorname{deg}(t)-\operatorname{cir}(r, s) \\
\quad=2 \operatorname{deg}(r)+2 \operatorname{deg}(s)+2 \sum_{t \in r-s \text { path }} \operatorname{deg}(t) \\
\leq 2(\text { Total degree of a graph })=2(2|\mathrm{E}|) .
\end{gathered}
$$

Hence $\operatorname{cir}^{D}(r, s)-\operatorname{cir}(r, s) \leq 4|E|$.

\section{CONCLUSION}

Graphs are of great utility to model a variety of relations and methods across various disciplines of science representing problems in a practical way. The various classes of graphs particularly on the distance, radius and diameter basing on theorems give valuable inputs to potential applications.

\section{REFERENCES:}

1. Buckley, F., Harary, F., Distance in graphs, Addison-Wesley, Longman, 1990.

2. Chartrand, G., Escuadro, H., Zhang, P., Detour distance in graphs, J. Combin. Comput 53 (2005), 75-94.

3. Chartrand, G., Zhang, P., Distance in graphs-taking the long view, AKCE J. Graphs Combin., $\quad 1$ (2004), 1-13.

4. Chartrand, G and P. Zang, Introduction to Graph Theory, Tata McGraw-Hill, (2006).

5. Reddy Babu, D., Varma, P.L.N., D - distance in graphs, Gold. Res. Thoughts, 2 (2013) 53-58.

6. Venkateswara Rao V., Varma P. L. N., Detour Distance in Graphs w.r.t. D-distance, Ponte International Journal of Sciences and Research. Res.73 (7) (2017) 19-28.

7. J. Veeranjaneyulu, P. L. N Varma, Circular D-distance and Path graphs, International Journal of Recent Technology and Engineering (IJRTE). Vol.7 (5S4) (2019) 219-223. 